\title{
Mathematical modeling of geophone magnetic ring for sensitivity studies
}

\author{
Mawa Patrick Luka a, Ser Lee Loh b, Dennis Ling Chuan Ching c, ${ }^{*}$ \\ a,c Universiti Teknologi PETRONAS ,Seri Iskandar, 32610 Perak, Malaysia \\ b Universiti Teknikal Malaysia Melaka, 76100 Durian Tunggal, Melaka Malaysia \\ * Corresponding author: dennis.ling@utp.edu.my
}

\section{Article history}

Received 7 November 2017

Accepted 8 November 2017

\begin{abstract}
Geophone (model SM-24) pertaining its performance to detect surface wave at Free Wave Generator is analyzed. Sensitivity studies have been conducted to model the physical disadvantage such as spring suspension and magnetic field in geophone. From mathematical model of the ring permanent magnet, applying "superposition" has suggested a solution: an external resistor across its output terminal to amplify the exhibited linear damping behavior. Geophone with $60 \%$, and $75 \%$ damping ratio are designed and built and the results show that $75 \%$ damping provide highest sensitivity. Analytic expression of vertical component of magnetic field shows that the damping ratio can be increased physically by increasing the radius of the magnetic field, however, by modifying the SM-24 damping ratio, similar outcome can be obtained.
\end{abstract}

Keywords: Sensitivity, resistivity, magnetic field

\section{INTRODUCTION}

A geophone is a device used by geologist to measure ground displacement by converting the detected motion into voltage based on Faraday's law of electromagnetic induction. Velocity sensing is critical requirements for geophone in high precision and diverse application in many industries. For example, in lithographic application, it is used in determining disturbance of payload caused by movement of the wafer scanner, further geophone is also found in application for accurate position of a complex lens system. Geophone is characterized by resonance frequency, which is recommended to be as low as possible in order to measure low frequency seismic activities.

Commercial geophone today exhibit a low resonance frequency, however not as low as below $4.5 \mathrm{~Hz}$, hence, high noise to signal is found at lower frequency. Another drawback of geophone is limited bandwidth $[1,2]$. Geophone which are configured with high resonance frequency often have high dimensions and required extra power supply, it turn not cost effective to be used in area where there is no sources of power and in application which required lengthy experimental time to acquire the required data such as Earth[3,4], respectively.

Magnetic spring model of geophone uses Halback configuration for vibration isolation structure [4]. Construction of a passive magnetic is bear by the used of ring magnet [6]. Difference between radial and axial magnetization gives a method of force calculation on ring magnets [7] and [8]. Based on Earnshaw's theorem, it indicates that "the object which are under the influence of field, will experience force applied in an inverse-square which relate to displacement", it does not exhibit property of stable levitation configuration [9] and [10]. Despite the Earnshow's theorem, the stability of passive magnet levitation is not possible, hence it was exempted. For example, the stability of magnetic levitation is possible base on diamagnetic material [11] and spin stabilization [12], or use of elastic materials, [13].
Geophones with $1 \mathrm{~Hz}$ resonance frequency are often very large. Commercial geophones today has limited bandwidth due it's the mechanical system (spring), and high damping factor at an open circuit (the output signal tend to oscillate in undesirable manner). Therefore, this article illustrated a study to improve total damping and sensitivity of geophone at low frequencies of $1 \mathrm{~Hz}$. Mathematical Model of Geophone Magnetic Ring is reviewed. Velocity potential is chosen, and possible enhancement such as "superposition" is suggested for possible sensitivity improvement. Design a circuit which will improve geophone output signal at lower frequency range of 1 to $8 \mathrm{~Hz}$ is the goal of this studies.

\section{METHODOLOGY}

\section{Semi Analytic Modeling}

Elementary principle of geophone is a mass suspended by mechanical springs. When a velocity in the $Z$-direction is applied, both the suspended mass/coil will oscillate proportional to the applied velocity. For frequencies below and above the resonance frequency, the oscillation of the mass is assumed constant.

Working principle is derived from Faraday's law;

$$
\nabla \times E=-\delta B \delta t
$$

where $\boldsymbol{E}$ stand for electric field strength and $\boldsymbol{B}$ is magnetic flux density. Since the magnetic flux is in a close surface, equation (1) can be rewritten in integral form

$$
\oint E . d l=-\delta t \int B . d s
$$

As a result, electromotive force $(e m f)$ is created by the magnetic field $[14,15]$. Geophones are constructed using cylindrical permanent magnet, as shown in the Figure 1. By solving the magneto-static field equation, analytical explanation gives the vector potential model. 


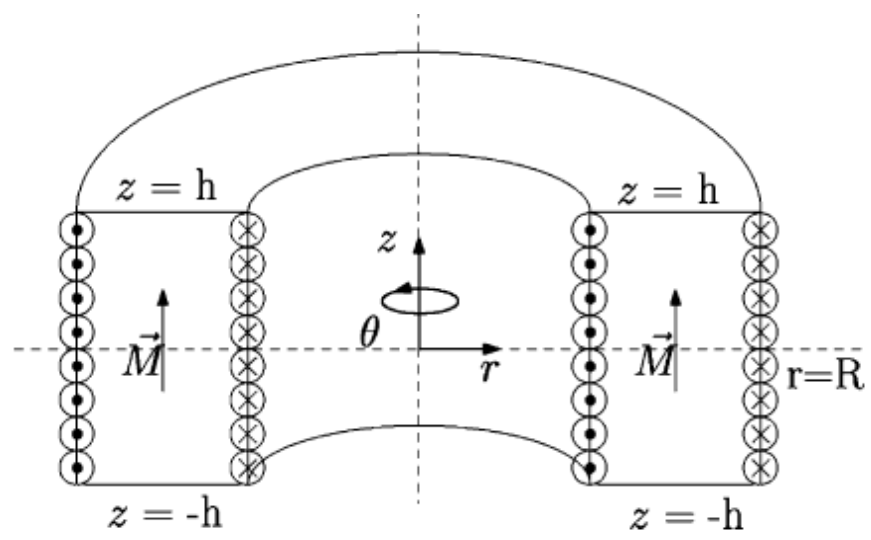

Fig. 1 Model of ring magnet [19]

Given

$$
B=\nabla \times A, \nabla \cdot B=0 \text { and } \nabla \times H=J
$$

$\boldsymbol{B}$ is magnetic flux density, $\boldsymbol{M}$ is the magnetic field, and $\boldsymbol{A}$ is the Vector potential $[16,17,18]$. Equations (2) can be expressed as

$$
B=\mu_{0}(H+M)
$$

By assuming infinite domain, where no boundaries and no free current or $J=0$ is then written as:

$$
A(r)=\frac{\mu_{0}}{4 \pi} \int \frac{J m\left(r_{\prime}\right)}{\left|r-r_{\prime}\right|} d r^{\prime}
$$

and current distribution

$$
J_{M}\left(r^{\prime}\right) d r^{\prime}=J_{M} \cos \theta^{\prime} R d \theta^{\prime} d z^{\prime}
$$

with vector different

$\left|r-r^{\prime}\right|=\sqrt{\left(r-R \cos \theta^{\prime}\right)^{2}+\left(-R \sin \theta^{\prime}\right)^{2}+\left(z-z^{\prime}\right)^{2}}$

$\theta^{\prime}$ is referred to phase lag due to current. Vector potential reads:

$$
A_{\theta}(r, z)=\frac{\mu_{0} J_{M}}{4 \pi} \int_{Z^{\prime}=h}^{h} \int_{\theta^{\prime}=0}^{2 \pi} p d \theta^{\prime} d z^{\prime}
$$

with

$$
p=\frac{R \cos \theta \prime}{\sqrt{\left(z-z^{\prime}\right)^{2}+R^{2}+r^{2}-2 R \cos \theta \prime}}
$$

\section{RESULTS AND DISCUSSIONS}

Geophone is regarded as electromagnetic voltage generator. Output is proportional to the rate of change of magnetic flux; output is not directly proportional to ground displacement, but its proportional to the velocity associated with the displacement. Therefore, geophone output is measured in volt $/(\mathrm{m} / \mathrm{sec})[20]$.

In this research, we proposed an explanation or hypothesis is written to show resistivity is related to vector potential (6) and magnetic surface current density $\left(J_{M}\right)$ of a geophone. Shunt resistor is used to filter the input and output current density. Both the output of amplitude and voltages are compared to observe and justify the hypothesis. Geophone (SM-24) has been selected. Three experimental results are recorded as following.

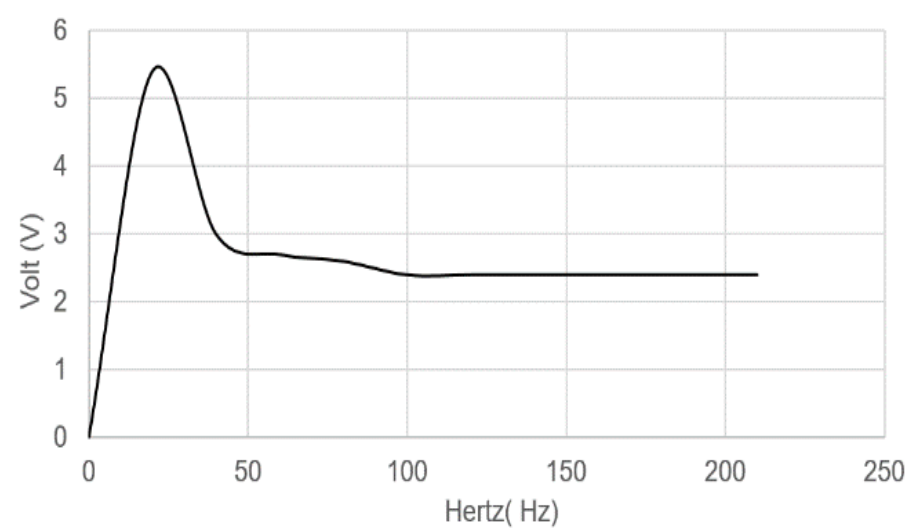

(a)

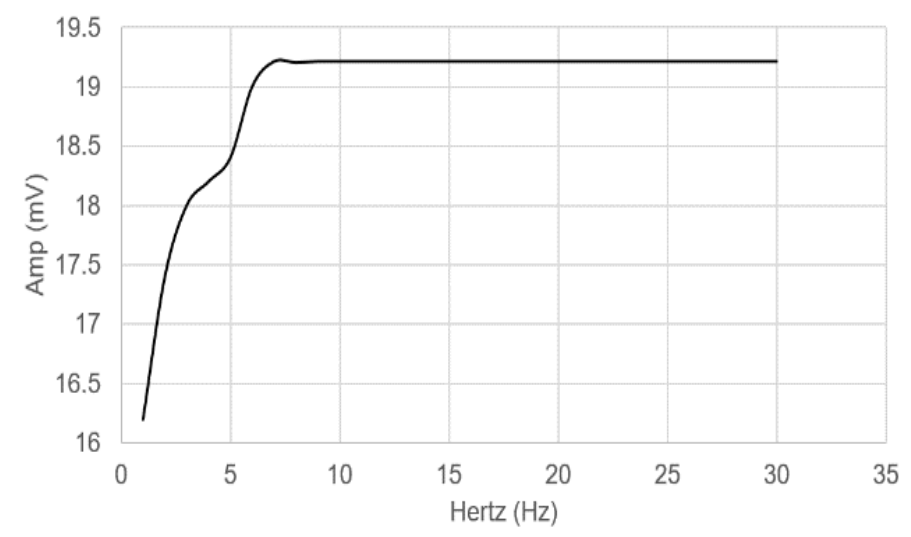

(b)

Fig. 2 (a) Output voltage of open circuit without shunt resistor. (b) Amplitude of open circuit without shunt resistor.

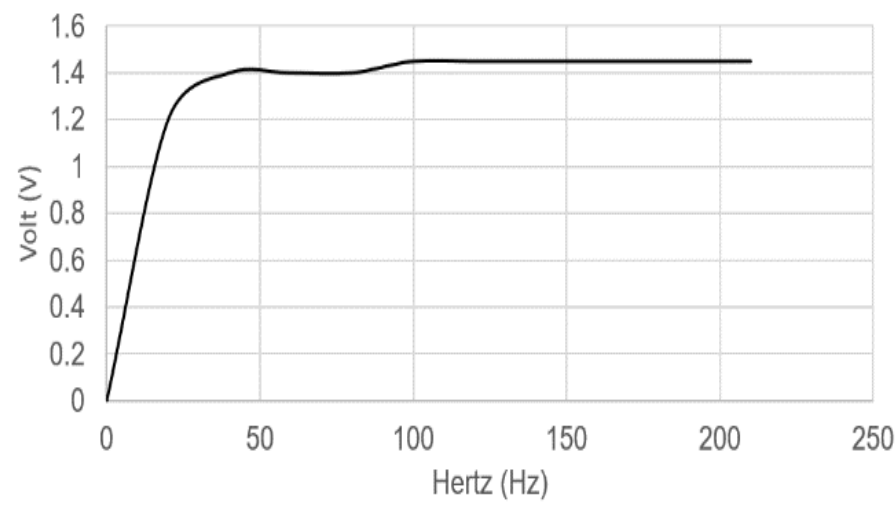

(a)

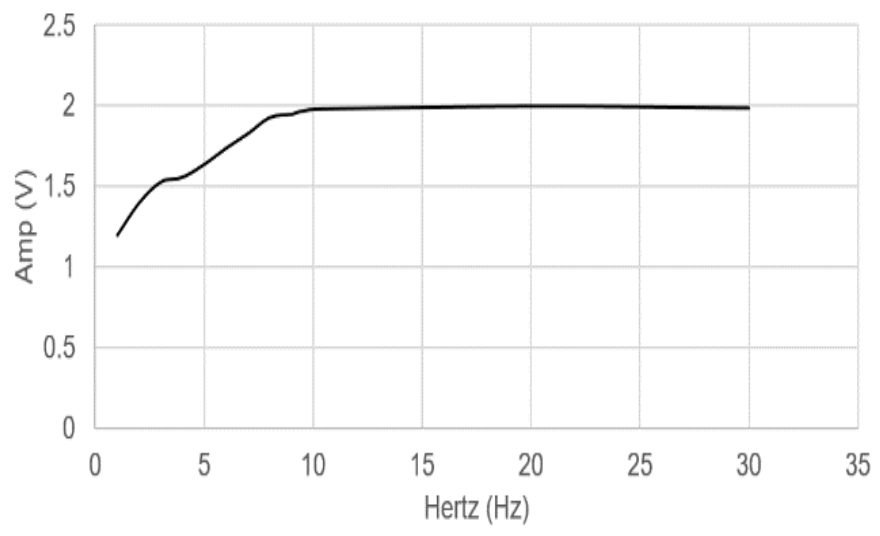

(b)

Fig. 3 (a) Output voltage of $500 \mathrm{Ohm}(\Omega)$ shunt resistor. (b) Amplitude of $500 \mathrm{Ohm}(\Omega)$ shunt resistor. 


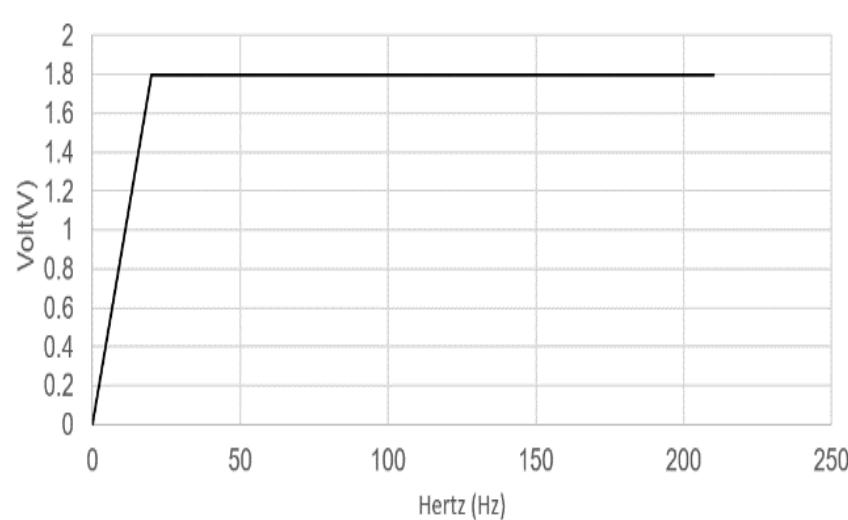

(a)

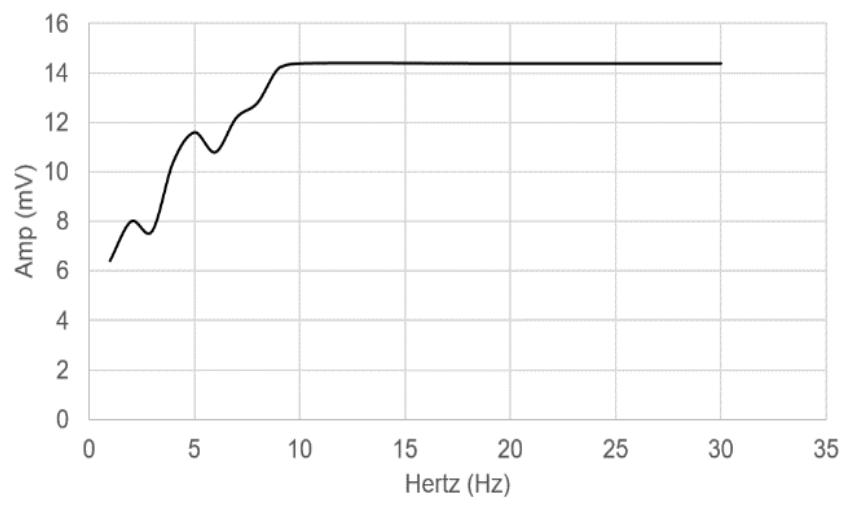

(b)

Fig. 4 (a) Output voltage of $1000 \mathrm{Ohm}(\Omega)$ shunt resistor. (b) Amplitude of $1000 \mathrm{Ohm}(\Omega)$ shunt resistor.

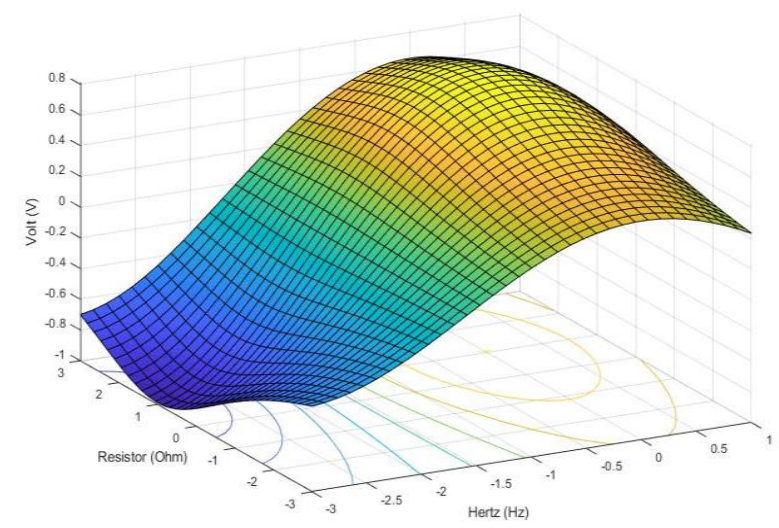

Fig. 5 Simulation of vector potential of a magnetic ring. Wave profile with amplitude from -3 to 1 hertz is plotted and related to resistivity (ohm) and output (volt).

Geophone motion is a force oscillatory motion, with resonant peak at the natural frequency. Manufacturers of commercial Geophone always specific the natural frequencies between open circuit and circuit with damping; underdamped, critical damped and overdamped. Figure 2 shows the underdamped output, while Figure 3 illustrated the critically damped output. Overdamped response is expressed in Figure 4.

When the geophone is overdamped, the unstable output is recorded in Figure 4(b). From Figure 2, 3 and 4, velocity potential of the geophone is said related to displacement of the coil and magnet. Shunt resistor acts as the resistivity filter or low-cut filter has attenuated the output as shown in Figure 2(a) and Figure 3(a). By adding shunt resistor linear damping is achieved in these experimental studies.

From experiment, high frequency gives the phase lag $0^{\circ}$ while at very low frequency $(1 \mathrm{~Hz})$ there is no relative displacement between the coil and magnet, and the phase lag is $180^{\circ}$, hereafter, recorded output voltage is zero. When natural frequency is referred to, the output is maximum at natural frequency. A simulation output is shown in Figure 5. At 1 Hertz, the voltage reading is 0 volt similar to the experiment result.

\section{CONCLUSION}

Geophone motion is a force oscillatory motion, with resonant peak at the natural frequency. To study total damping and sensitivity of geophone at low frequencies, it's important to obtain the critical and overdamped frequency of a designed geophone. Mathematical representation of a velocity potential suggests that the phase lag, resistivity, surface current density should be analyzed to obtain the reference natural frequency. Sensitivity will increase dramatically when damping ratio increases.

\section{ACKNOWLEDGEMENT}

Authors will like to thanks funding agents (STIRF FUND, Universiti Teknologi PETRONAS and MOHE FRGS 0153AB K78) to support the researches.

\section{REFERENCES}

Robertson, W. (2003). Design of a non-contact magnetic spring for vibration isolation. The University of Adelaide, Australia: Research proposal.

Barzilai, A. (2000). Improving a geophone to produce an affordable, broadband seismometer. Stanford University: PhD Thesis.

[3] Braunbek, W. V. (1939). Fresichwebende Korper im elektrischen und magnetisachen feld. Tubingen.

[4] Cathey, J. J. (2001). Electric Machines. McGraw-Hill.

[5] Cedrat Groupe. Accessed in 2008 from www.cedrat.com.

[6] Delamare, J., Yonnet, J. P. and Rulliere, E. (1994). A compact magnetic suspension with only one axis control. IEEE Trans. Magn. $30(6)$

[7] Earnshaw, S. (1842). On the nature of the molecular force which regulate the constitution of the luminferous ether. Trans. Cambr. Philos. Soc.797-112

[8] Filatov, A. V. and Maslen, E. H. (2001). Passive magnetic bearing for flywheel energy storage systems. IEEE Trans. Magn. 37 (6).

[9] Florian, E. F., Haggard, S. E. and Riley, T.E. (1979). Geohone with shaped magnetic field. Marks Product Incorporated: UK Patent Application, UK 2022359.

[10] Furlani, E. P. (2001). Permanent magnet and electromechanical devices. Academic Press.

[11] Geospace. Accessed in 2008 from www.geospace.com.

[12] Hol, S. A. J. Design and Optimization of a magnetic gravity compensator. Eindhoven University of Technology: Ph.D. Thesis. 155-157.

[13] ION Sensor. Accessed in 2008 from www.geophone.com.

[14] Jones, W. (1980). Earnshaws theorem and the stability of matter. Eur. J. Phys. 85-88.

[15] Magnetic innovations.www.magneticinnovations.com, accessed in 2008 .

[16] Nagaya, K. and Ishikawa, M. (1995). A noncontact permanent magnet levitation table with electromagnetic control and its vibration isolation method using direct disturbance canellation combining optimal regulators. IEEE Trans. Magn. 31 (1).

[17] Oome, A. J. J. A., Janssen, J. L. G., Encica, L., Lomonova, E., and Dams, J. A. A. T. (2009). Modeling of an electromagnetic geophone with passive magnetic spring. Sensors and Actuators A: Physical 153(2). 142-154

[18] Palm, W. J. and Modeling. (1999). Analysis and control of dynamic systems. John Wiley and Sons Inc.: Second edition.

[19] Ravaud, R., Lemarquand, G., Lemarquand, V., and Depollier, C. (2008). Analytical calculation of the magnetic field created by permanent-magnet rings. IEEE Trans.Magn.44 (8). 1982-1989.

[20] Recdeker, M. (1984). Geophone. Mobil Oil Corporation: US Patent Application, US 4,597,070. 